\title{
La psicología fenomenológica de Husserl y la psicopatología
}

\section{Husserl's phenomenological psychology and psychopathology}

\author{
Gustavo Figueroa C. ${ }^{1}$
}

\begin{abstract}
Background. Edmund Husserl's Phenomenological psychology is a discipline set to play an important role in the empirical phenomenological psychopathology. Objective. To focus on the basic philosophical and psychological assumptions of Phenomenological psychology. Method. To inquire into Husserl's interpretations on the nature of Phenomenological psychology in order to understand the presuppositions of phenomenological psychopathology in psychiatry. Results. Phenomenological psychology is described as an aprioric, eidetic, intuitive, purely descriptive, and intentional science of the psychical, which remains entirely within the realm of the natural attitude Conclusions. Phenomenological psychology is destined to supply the essential insights needed to give meaning to the research presented under the title of psychopathological phenomenology. Key words. Husserl, phenomenological psychopathology, phenomenology
\end{abstract}

Key words: Husserl, phenomenological psychopathology, phenomenology.

Rev Chil Neuro-Psiquiat 2008; 46 (3): 224-237

$\mathrm{P}$ ronto se cumplirá un siglo desde que Jaspers introdujo por vez primera el método fenomenológico en la psicopatología ${ }^{1}$. Su inmediata aceptación y difusión al interior de la psiquiatría, especialmente alemana, hizo suponer que se estaba ante un giro revolucionario en la concepción de la psicopatología, hasta entonces dominada por la escuela francesa, que había sostenido que sus raíces estaban en la clínica sin preguntarse por la naturaleza de su método. De una manera apresurada se afirmó que el innovador libro de Jaspers "Psicopatología General”, publicado al año siguiente, representaba un ejemplo indesmentible de que la fenomenología psiquiátrica iba a transformarse en la base firme donde edificar toda la psicopatología científica moderna ${ }^{2}$. Cuando la fenomenología conquistó al resto de Europa y luego a Estados Unidos, quedó aún más firmemente establecido que su aplicación en la clínica y la investigación eran garantías de cientificidad y rigor metodológico ${ }^{3-6}$.

Jaspers fue el primero en rechazar con particular energía la afirmación de que la fenomenología constituía el fundamento último de la psicopatología, porque aquella representaba sólo un método, entre varios otros existentes ${ }^{7}$. Sin embargo, las críticas más feroces provinieron de investigadores interesados por la validez y confiabilidad de los hallazgos clínicos. Ellos atacaron implacable y demoledoramente todo estudio que

Recibido: 15 de enero de 2008

Aceptado: 2 de agosto de 2008

1 Departamento de Psiquiatría. Escuela de Medicina, Universidad de Valparaíso. 
no estuviera centrado en lo objetivamente observable, el positivismo experimental y el positivismo operacionalista, es decir, que no acatara las estrictas reglas que Hempel había decretado con claridad $^{8-10}$. Producto de esta inversión radical, la psicopatología sufrió una profunda crisis de sus fundamentos que estalló en la psiquiatría a comienzos de la década del 70, y culminó con la psiquiatría basada en la evidencia, la elaboración del DSM-III y el nacimiento de la neurobiología de la mente ${ }^{11-19}$.

La fenomenología perdió todo el crédito anterior y, en el mejor de los casos, se la aceptó condescendientemente como un residuo histórico, anticuado y conceptualmente superado. Continuó usándosela en la clínica cotidiana, casi de modo furtivo, pero bajo la vaga, confusa y ambigua denominación de "psicopatología descriptiva" ${ }^{20}$. Vale decir, el cuestionamiento positivista no sólo arrasó con el método sino que fabricó una etiqueta "fenomenología"-que, evidentemente, no fue resultado de una investigación rigurosa sobre sus fundamentos teóricos y científicos, $\mathrm{y}$, menos aún, de sus principios filosóficos ${ }^{21}$. En una frase, fue consecuencia de sus presupuestos y prejuicios como ciencia natural antes que de un análisis estricto de los hallazgos fenomenológicos ${ }^{22}$.

Pero hay más todavía, y que no viene precisamente de los detractores declarados. A pesar de su rica productividad en calidad y cantidad, esta producción casi siempre estuvo basada en interpretaciones ligeras o errores conceptuales importantes por parte de los mismos investigadores que han practicado la fenomenología en la clínica. Los motivos de estas equivocaciones son múltiples y sus alcances han sido trascendentes para la psiquiatría ${ }^{6,23-26}$.

El presente trabajo se reducirá a una doble meta como primer paso para ayudar a solucionar esta paradójica historia de la fenomenología al interior de la ciencia psiquiátrica. Por un lado, mostrar brevemente que la "Fenomenología" de Jaspers reposa en un malentendido de principio, aunque sea un malentendido productivo. Por otro, se recurrirá a investigar la "Psicología feno- menológica" de Husserl, puesto que es la fuente originaria y fundamento de la fenomenología en psicopatología.

\section{Un malentendido productivo}

La definición de Jaspers es bien conocida y no varió en lo esencial con los años. "La fenomenología nos dice Jaspers -tiene la tarea de hacernos presente intuitivamente (anschaulich vergegenwärtigen) aquello que los pacientes realmente vivencian, considerarlo de acuerdo a sus relaciones de parentesco, delimitarlo, diferenciarlo lo más claramente posible y entregarlo en términos precisos". Y agrega que "ya que la vida psíquica ajena al igual que la física nunca puede ser percibida directamente, se trata siempre de un hacer presente, de un empatizar (Einfühlen), de un comprender (Verstehen)". O sea, "estamos entregados al «juicio psicológico» de los enfermos....Ellos mismos son los observadores"7. Y agrega, en una significativa nota a pie de página, que se corresponde con la que inicialmente Husserl denominó "Psicología descriptiva" de las "manifestaciones de la conciencia". Esto es, Jaspers acepta que su método proviene de Husserl pero que se aparta por completo de su fenomenología posterior, especialmente la relacionada con la "intuición de esencias", porque el suyo es un "procedimiento empírico".

Las características de la fenomenología para Jaspers son claras aunque difíciles de llevar a cabo o ejecutar porque la existencia psicológica subjetiva es altamente compleja.

1. Es un método o modo de acceder a la psicopatología del paciente y, como tal, debe actuar en armonía o complementación con otros métodos o caminos de investigación. Vale decir, lo psicopatológico trasciende la fenomenología porque la vida psíquica enferma es una totalidad complejísima y que como tal se expresa de muy diferentes maneras. Lo cual supone límites muy precisos para la fenomenología que ella no debe sobrepasar, pero también ha de defenderse de intromisiones foráneas que amenazan con igno- 
rar o menospreciar su especificidad (ej. aseverar que la estructura de una alucinación se reduce a un desorden bioquímico cerebral localizado).

2. Es un procedimiento atenido a hechos, a lo realmente existente aunque se oriente a objetos que no se muestran sensorialmente ante los ojos. No es una descripción como la de las ciencias naturales pero el principio lógico empleado es el mismo. En síntesis, es ciencia empírica, y como tal está referida a la experiencia inmediata o fáctica, pero sin la presencia efectiva o real del objeto.

3. Su propiedad distintiva consiste en la descripción. Con rigor describe de acuerdo a categorías sistemáticas y comparaciones contrastantes $\mathrm{y}$, aunque se restringe a fenómenos aislados o únicos, puede abarcar totalidades como la conciencia psicológica. Pero hay que ser cuidadoso. La descripción rigurosa es altamente compleja: diferencia, delimita, confronta, pone en relación, señala, comprueba, fija en términos exactos, unívocos y puntuales sus hallazgos.

4. Su materia son las vivencias psíquicas individuales, los estados psicológicos positivamente vivenciados por el paciente, lo que sucede en su interioridad con todos sus detalles, matices, modulaciones y formas especiales. Esto significa que, además de "qué" vivencia, importa "cómo" lo vive, "cómo" lo expresa, "cómo" coloca el acento enfrente del interlocutor. A modo de ejemplo recordemos que las histéricas comunican su interioridad con un dejo de poca confiabilidad, de modo que esta autodescripción suscita una indesmentible atmósfera de incredulidad y sospecha. Esto es, se puede poner en tela de juicio el contenido de lo expresado pero no su carácter ambiguo o dudoso.

5. La postura fenomenológica (Einstellung) es una actitud muy peculiar que debe asumir activamente el psicopatólogo. Significa dejar de lado toda teoría recibida por justificada que parezca científicamente, abstenerse de elaborar hipótesis o emitir interpretaciones, no considerar los juicios que se hayan sostenido con anterioridad, no hacerse eco del sentido común. O sea, limitarse a lo dado realmente en la conciencia y exclusiva- mente a lo dado. Esta deliberada ausencia de prejuicios fenomenológicos no es una posesión que ostenta el investigador, lo contrario, es un legado que se gana y debe ser reconquistado en cada oportunidad con esfuerzo y dedicación extrema gracias a un tenerlo como meta consciente y permanentemente.

6. Las vivencias de la conciencia son captadas por medio de intuiciones (Anschauungen) o visiones pregnantes que se adentran en los fenómenos inmediatamente experimentados. Este ver no es naturalmente un ver sensorial sino comprensivo, que penetra hasta lo último (Letzte) de la vivencia, esto es, lo distintivo, específico y peculiar a ella y sólo a ella. Se alcanza lo "fenomenológicamente último" cuando se llega a lo irreductible, a lo que no puede irse más allá porque se ha cogido su núcleo fundamental (ej. se aprehende el delirio primario cuando se alcanza su apodicticidad e inderivabilidad como totalmente diferentes e inconfundibles con la de las ideas deliroides, sin posibilidad de transiciones o fenómenos intermedios porque se visualiza intuitivamente su estructura última y radical).

\section{La fenomenología como ciencia estricta}

Husserl fundó la fenomenología al comenzar el siglo XX, pero no se interesó por la psicopatología ${ }^{27-29}$. Sin embargo, recibió a Jaspers cuando éste era un principiante y lo alentó a continuar en sus esfuerzos pioneros en este campo. Jaspers fue muy duro en su apreciación del filósofo a pesar de la amable acogida. Lo criticó con violencia aseverando que Husserl pregonaba que iba hacia los fenómenos en si pero que esta era sólo una postura, ya que no se interesaba realmente en alcanzarlos. Él se quedaba atascado en las palabras y enunciados programáticos sin poner en marcha real su método de "a las cosas mismas" 30 .

Quizás Jaspers no quiso entender bien que el fundamento inicial de la fenomenología de Husserl era sustancialmente diferente al suyo. Era filosofía y además radical. Husserl arranca de una 
situación que le parece históricamente irrefutable. Desde Descartes la filosofía ha buscado partir de algún hecho o estado de cosas (Sachverhalt) que sea en si innegable, que se presente ante nuestros ojos como absolutamente incuestionable, que se muestre como evidente de suyo, aunque no sea muy importante o teóricamente elaborado. Pues bien, resulta palmario que no lo ha conseguido hasta ahora y todos los sistemas filosóficos han fracasado sucesivamente producto de la falta de evidencia de sus principios primeros ${ }^{31}$. Mientras las ciencias naturales han avanzado y se han desarrollado con paso seguro desde el renacimiento, de modo que cada hallazgo representa un nivel superior que se suma a los anteriores, en filosofía no ha acaecido lo mismo. Sus teorías representan opiniones que son inmediata o posteriormente refutadas, de modo que nos encontramos sin un efectivo progreso sino con una acumulación desordenada de visiones de mundo (Weltanschauungen), con una sabiduría milenaria desperdigada aunque útil posiblemente para la conducción de la existencia cotidiana o con un historicismo que es un relativismo o escepticismo disfrazado $^{32}$.

Esto quiere decir que la filosofía no ha conseguido establecerse como una ciencia estricta (streng), ni siquiera como ciencia imperfecta, porque sus enunciados teóricos no exponen hechos objetivos, válidos y comprobables. Esta es la meta de Husserl y la que dirige toda su reforma del pensamiento filosófico. Transformar la filosofía en ciencia estricta, o, como lo había expuesto explícitamente Kant con anterioridad, penetrar por fin en el seguro camino de la ciencia ${ }^{33}$. La elaboración de la fenomenología pura será la base firme para su empresa de fundamentación ${ }^{34}$. De ahí que para él, "el impulso primario para la investigación [filosófica] no tiene que provenir de las [otras] filosofías, sino de las cosas mismas (Sachen selbst) y sus problemas" ${ }^{31}$.

Dicho de manera resumida. La fenomenología pretende ser una ciencia absoluta, donde absoluta significa que su conocimiento 1) debe caer sobre un objeto o estado de cosas que sea disponible por todos sin discusión posible, acce- sible sin ningún género de dudas, y 2) que al mismo tiempo sobre este objeto o estado de cosas se deben ir teniendo conocimientos justificados con evidencia plena por la condición inherente o propia de él.

Lo que Husserl ambiciona es que el objeto (Sache) mismo esté presente. Puede que esté "inmediatamente" presente, por ejemplo, cuando tengo ante mí el cuadro que acabo de ver hace unos instantes en una exposición, o sea, en la memoria reciente. No es la única forma, ya que puede estar "mediatamente", como cuando, al ver una fotografía de mi hermana, ella está presente aunque de manera oblicua. Sin embargo lo buscado por Husserl es otra manera de mediatez o inmediatez: es que esté "originariamente" dado. Es el objeto o estado de cosas presente "en carne y hueso" (leibhaftig), real o corporalmente, presencia plena o cumplida (erfüllt) $)^{35}$.

\section{Fenomenología y psicología fenomenológica}

Quedan claras las discrepancias de principio entre Jaspers y Husserl. Mientras Jaspers se contenta con elaborar un método empírico al interior de la ciencia de la psicopatología describiendo rigurosamente las vivencias subjetivas de los enfermos, Husserl pretende fundar una filosofía primera que parta de hechos absolutos con validez incondicional, esto es, una ciencia estricta.

Para Jaspers es la diferencia entre ciencia y filosofía. Él piensa que Husserl las confunde en lugar de señalar con precisión sus barreras infranqueables. Como ciencia, la psicopatología es un conocimiento necesario, metódico y válido: un saber cómo, por qué razones, en qué límites y en qué sentido -lo exacto (richtig). La filosofía, por el contrario, es un sistema poseedor de una certidumbre absoluta, ahora o en el futuro, aclara los fundamentos y tiende a la unidad total posee la verdad (Wahrheit). O sea, lo válido e impositivo frente a lo absoluto e integral ${ }^{36}$.

Contrariamente Husserl busca el saber propio de la ciencia. Las ciencias de la naturaleza y 
las ciencias del espíritu ya iniciaron su camino hacia el conocimiento de las cosas. Todavía les falta la ciencia primera o filosofía que les sirva de principio o base originaria. La filosofía es esa ciencia estricta, la indeclinable aspiración del hombre a un conocimiento puro, inconmovible y absoluto. La única filosofía que encarna y persigue ese ideal de pureza es la fenomenología.

Para Jaspers, "fenomenologia" es una ciencia empírica, y, para Husserl, es una ciencia primera o filosofía. Pero además la fenomenología de Husserl se ocupa de un tema muy particular que tiene especial importancia para la psicopatología. Lo que sucede es que, para Husserl, la fenomenología estudia la "conciencia" y los "fenómenos" que aparecen en ella.

Descartes hizo de la duda su método porque fue el primero en buscar la certeza como criterio de verdad. Su solución frente al poner en entredicho toda la realidad es conocida. La única realidad que se salva de la duda metódica es el yo pensante mismo: pensamiento y realidad del ego $^{37}$. Husserl ve que Descartes yerra desde el inicio mismo aunque admira sus sobresalientes méritos. Todo pensamiento (cogitatio), aunque se ponga completamente en duda su verdad, es un pensamiento de algo (cogitatum) y este "algo" es un objeto muy particular. Descartes pasa por alto esta peculiarísima cualidad del "algo" y se concentra en el yo. No repara en que la fórmula precisa para su hallazgo es ego cogito cogitatum, yo pienso pensamientos. Pensar los pensamientos o "algos" es una correlación. Y a esta correlación se va a dedicar la crítica de Husserl y esta crítica lo conducirá a decir que el cogitatum o pensamiento o "algo" en cuanto tal es lo que se debe llamar "fenómeno"38.

Fenómeno es lo que es manifiesto en tanto en cuanto es manifiesto y tal como es manifiesto, como lo expresa su etimología de phainómenon. Lo pensado en el pensamiento o "algo" o cogitatum es pura y simplemente fenómeno. Esto pensado puede reacaer tanto sobre el mundo exterior como sobre mis propias vivencias internas o estados psicológicos.

A su vez, todo pensamiento o cogitatum, en tanto manifestación de algo, se manifiesta a alguien. Aquella ante quien el pensamiento es fenómeno Husserl la designa conciencia. Fenómeno y conciencia son, pues, términos correlativos. Toda conciencia es conciencia de algo, y este algo es el fenómeno que se da en aquella conciencia ${ }^{39}$.

La fenomenología estudia los fenómenos que se muestran en la conciencia, vale decir, reduce toda realidad a puro fenómeno en cuanto aparece como tal fenómeno en la conciencia. Pero así como fenómeno no es apariencia subjetiva, así tampoco conciencia es un estado subjetivo. La correlación fenómeno-conciencia está por encima de cualquier dualismo sujeto-objeto.

Resumamos. La fenomenología para Jaspers es una ciencia empírica de las manifestaciones subjetivas de la vida psíquica patológica, para Husserl es filosofía absoluta de los fenómenos de la conciencia pura. Se puede sospechar con razón que ambas concepciones, a pesar de sus radicales diferencias, tienen muchos asuntos o problemas en común (conciencia, fenómeno psíquico, vivencia, ego, etc). Husserl no investigó estas similitudes ni divergencias porque, como ya se dijo, no se ocupó personalmente de la psicopatología, pero si lo hizo con la psicología. La "Psicología fenomenológica" es su respuesta a la relación íntima entre fenomenología como ciencia filosófica estricta y fenomenología como ciencia empírica de lo psíquico. Lo que significa a su vez que la fenomenología de Jaspers corresponde a un caso especial de ciencia empírica de lo psíquico: es lo psíquico pero anormal. O sea, si entendemos la relación que Husserl tuvo con lo psicológico entenderemos como corolario como puede fundamentar la ciencia de la psicopatología.

\section{La psicología empírica}

Desde las Investigaciones lógicas hasta La crisis de las ciencias europeas y la fenomenología trascendental, esto es, desde 1900 hasta su muerte, Husserl se interesó intensa y progresivamente por las relaciones entre Fenomenología y Psicología ${ }^{40}$. No por casualidad ni por erudición. La fenome- 
nología fue pasando por etapas y cada una de ellas representó una profundización de sus exigencias de pureza y certeza en referencia a la psicología de la conciencia y sus contenidos. En una frase, parte desde lo psicológico tal como se da en la vida cotidiana para alcanzar gradualmente la psicología positiva y llegar por fin a lo psíquico puro ${ }^{41}$.

Husserl recurrió a la psicología empírica para tratar de descubrir una respuesta a sus investigaciones sobre los "fenómenos" que se muestran en las vivencias, pero se tropezó con que aquella lo desviaba precisamente por sus graves prejuicios que estaban a su base. La psicología empírica es 1) naturalista, supone de partida, antes de cualquiera prueba, que todo lo que aparece, incluído el hecho psíquico, es naturaleza física, "tiene lugar en el espacio y el tiempo de la naturaleza”, lo que conduce finalmente a la naturalización de la conciencia y sus vivencias; 2) ha vivido "en el espejismo de poseer un método científico-natural inspirado en el modelo físico-químico"; esto es, en lugar de adaptar el método a la índole propia del objeto en estudio lo psicológico, la investigación lo somete, obliga y constriñe a sus prejuicios físico-químicos, a su objetivismo a ultranza y a su matematización extremosa, en último término, a cosificar la conciencia y sus contenidos; y 3) demuestra una "ceguera ante lo psicológico" -"la homologación ingenua de lo dado en los datos de la experiencia psicológica con los de la experiencia corporal conduce a la concepción de los mismos como átomos o complejos de átomos y al paralelismo de tareas en ambos lados" ${ }^{\prime 2}$ (Tabla 1).

Husserl reconoce las cualidades indiscutibles de la psicología empírica y positiva. Lo que le critica sin desmayo es que no entrega, ni podrá entregar jamás en el futuro, por su propia condición de ciencia natural, lo específico de todo fenómeno de conciencia, la esencia de la vivencia del ego. Este reparo es de peso porque es precisamente lo que busca la fenomenología para constituirse en ciencia estricta. Esto quiere decir que la "Psicología fenomenológica" no es sin más una psicología científica positiva refinada, es un salto cualitativo, y, por ello, requiere ser diferenciada de ésta de manera rigurosa ${ }^{44}$.

\section{La psicología fenomenológica}

Entre 1925 y 1928, Husserl dedicó sus lecciones universitarias a exponer y desarrollar lo que bautizó como "Psicología fenomenológica" en sentido exacto. Han sido ignoradas hasta hace poco porque se publicaron muchos años después de su muerte y no han sido traducidas hasta hoy ${ }^{42}$. Lo que implica a su vez que fueron desconocidas no sólo por Jaspers sino por la mayoría de los investigadores que han aplicado y aplican la fenomenología en la psicopatología. Este motivo da cuenta, en parte al menos, lo afirmado al comienzo, de que los psicopatólogos han interpretado mal o seguido de manera insuficiente los exigentes procedimientos y conceptos elaborados por Husserl.

La Psicología fenomenológica pretende tener una relación con la Psicología empírica como la física pura la tiene con la física. Como psicología pura, su primera tarea es establecer los principios universales y necesarios que van a servir de

Tabla 1. Características de la psicología científica $\left.{ }^{*}\right)$

- Experimentación como método privilegiado

- Explicación de los fenómenos de la conciencia

- Estructuras predominan sobre acciones y actividades

- Atomismo de lo psicológico que desemboca en funciones aisladas

- Relación asociativa causal entre los átomos psíquicos

- Mecanicismo de las estructuras psíquicas y sus funciones

- Conciencia como resultado de las estructuras físicoquímicas subyacentes

- Uso de hipótesis constructivas que trascienden lo dado

- Monismo materialista

${ }^{\star}$ Modificada de: Pinillos JL. La psicología fenomenológica (1970) 
fundamento de la psicología positiva. Y para cumplir esta meta ha de partir del fenómeno de la intencionalidad de la conciencia, que ha sido sistemáticamente inadvertida aunque Brentano la introdujo por vez primera hace casi un siglo y medio $^{45}$.

No se trata de los mecanismos que son propios a la conciencia, mecanismos por los cuales tengo conciencia, sino de la conciencia misma, mejor aún, de la esencia de la conciencia ${ }^{46}$. Lo que sucede es que la conciencia no se define como función, o como todo momentáneo del vivenciar psíquico, o como la forma superior de adaptación del animal hombre al medio ambiente, o como darse cuenta de su entorno y de sí mismo, o como cualidad emergente del cerebro ${ }^{47-53}$.

Brentano señaló que los fenómenos psíquicos se diferenciaban de forma absoluta de los fenómenos físicos porque aquellos son intencionales. Usando la palabra en el sentido estricto de la escolástica medieval, intencionalidad de un objeto significa la referencia a un contenido, la dirección hacia un objeto, un mero "darse cuenta" de algo. La conciencia es siempre y sólo "conciencia-de", algo que sólo lo es "de" otro algo, una intentio -la conciencia envuelve intrínsecamente la "existencia intencional" de su objeto, aunque no la "existencia real" o realidad entendida como exterioridad independiente del sujeto.

Husserl está de acuerdo con el momento "de". Así, todo percibir es un "percibir-de", todo recordar es un "recordarse-de", todo imaginar es un "imaginarse-de". Es la intencionalidad como mera correlación o referencia. Pero cualquier "algo" de la conciencia no es un "contenido" sino exclusivo "término" intencional de la conciencia, algo que es manifiesto en ella, algo que aparece en ella, pero que no es ella misma ni parte de ella. Mi escritorio donde escribo, mi recuerdo de mis compañeros de infancia, un cuadrado imaginado no forman parte de mi conciencia. Aquí Husserl recuerda su puntualización hecha más arriba. Toda conciencia es conciencia-de un fenómeno, y todo fenómeno sólo puede ser lo que es como término o referencia de la conciencia. Por decirlo así, no existe una especie de recipien- te en cuyo interior se van acumulando las vivencias como cosas corpóreas. Con una frase algo novelesca, Sartre dice que, gracias a la intencionalidad, nos hemos liberado de "la vida interior", "todo está afuera, todo, inclusive nosotros mismos: fuera, en el mundo, entre los demás" ${ }^{4}$. Esta unidad intrínseca además tiene una característica peculiar. Es una unidad de "sentido": la intencionalidad abre el sentido de lo percibido, recordado, imaginado, padecido, etc.

Ahora se entiende mejor que se está buscando la esencia (Wesen) de lo psíquico, no simplemente sus atributos básicos, o reglas generales, o leyes empíricas, o enunciados válidos para cualquier sujeto concreto en alguna parte del mundo. La esencia es aquello que una cosa "es". Esencia es el ser de las cosas: ser percepción, ser imaginación, ser recuerdo, ser emoción. Frente al saber de "hechos" o positivo, el saber de "esencias" o puro es un saber sobre lo que constituye el metro o patrón de la realidad, en nuestro caso, la medida de lo psíquico. Para Husserl es determinante, porque la esencia de lo psíquico justifica racional y críticamente (kritische Rechtfertigung) el hecho psíquico no es un mera justificación empírica.

Para alcanzar las esencias Husserl recurre a la técnica de la variación imaginaria. Tomemos como ejemplo un objeto coloreado. Si es imposible conservar el color sin la superficie, es porque pertenece a la esencia del color no aparecer sin la superficie. Esto es, la técnica de la variación descubre las notas invariables, o sea, esenciales. Lo que no puede omitirse sin destruir el objeto, pertenece a su esencia

¿Basta con esto? Husserl piensa que no. Si su Psicología fenomenológica aspira a ser frente a la Psicología empírica una especie de matemática pura en relación a las matemáticas, él tiene que llevar a cabo un giro o vuelco. Este giro es de tal naturaleza novedoso y contrario al sentido común, que tendrá que acudir a explicarlo con particular detención en sus Conferencias de Amsterdam y de Paris de 1928 y 1931 respectivamen$\mathrm{te}^{38,42}$.

"La reducción psicologico-fenomenológica" 
consiste en poner entre paréntesis (Einklammerung) -epoché en griego, detención, suspensión†o suspender todo lo que se presenta ante mí como un hecho real o fáctico o existente del mundo. No se abandona la vida, se queda dentro de ella en toda su riqueza y detalle, pero cesa su vigencia en cuanto a considerarla real, se reduce a algo que no es real, no interesa ya en creer si está o no efectivamente afuera. La creencia universal de que el mundo "simplemente existe en realidad" (einfach da-seiend), se suspende ¿A qué queda reducido el mundo? Como ya se dijo, a todo lo que aparece en la conciencia psicológica y en tanto aparece a la conciencia psicológica. En otros términos, surge la esfera de lo puramente psicológico, lo psicológico en toda su pureza y, por tanto, desprovisto de la características fácticas, contingentes y existenciales que le pertenecen al sujeto como organismo viviente. Así, se excluye o se prescinde de la totalidad de los elementos psico-físicos y sus componentes corporales reales con los que lo psíquico está esencialmente conectado ${ }^{55-57}$.

Por tanto, la Psicología fenomenológica es psicología, pero "pura", porque ha eliminado metódicamente todo el ámbito de la experiencia corriente, las realidades psico-físicas y corporales acompañantes, las teorías científicas vigentes, los hechos aislados o particulares y las generalidades empíricas que pueden ser derivadas de ellas. Al igual que la física teórica, su exactitud e incondicionalidad provienen de la elaboración de un sistema de formas a priori característico de cualquier acto psíquico como tal.

La Psicología fenomenológica es producto de un considerable esfuerzo intelectual y científico, que se obtiene por tanto "reduciendo" (purificando) progresivamente los fenómenos que se muestran en nuestro mundo cotidiano o mundo-de-la-vida en el que creemos con fe ciega (Urglaube) y en el cual vivimos ${ }^{58-60}$. Las características definitorias se pueden resumir en el siguiente esquema ${ }^{42}$.

1) A priori: Significa que se circunscribe a todo aquello sin lo cual el ser y la vida psicológicas serían impensables como tal vida psicológica.

2) Eidética: Su meta son las necesidades esen- ciales y generalidades esenciales de la conciencia y los fenómenos conscientes. Sólo en segundo término o posteriormente se dirige al aclaramiento de la facticidad psicológica y a las teorías psicológicas positivas.

3) Intuición: Es la fuente del a priori. Son visiones originales inmediatas, que se deben aceptar, pero sólo dentro de los márgenes en las cuales aparecen. Gracias a estas visiones o intuiciones internas (Innenschau), al análisis de lo intuido $\mathrm{y}$ al ascenso intuitivo hacia necesidades generales, se obtienen determinaciones que son determinaciones de la esencia de lo visto.

4) Descripción: No pre-supone nada sino se ciñe estrictamente a lo visto y a fijarlo en expresiones claras, completas y unívocas.

5) Intencional: Carácter esencial del ser psíquico y de la vida psíquica. La vida intencional es vida consciente y conciencia es conciencia-de algo.

6) Postura o actitud dogmática natural: Aunque se acepte la reducción psicologico-fenomenológica se permanece en el mundo real y objetivo; mejor aún, su objeto es el mundo natural objetivo aunque se ha suspendido la creencia en su existencia externa. No se cuestiona a aquel, se lo acepta como fenómeno y no se pregunta a la conciencia cómo puede hacer que este aparezca o se constituya como en la "fenomenología constitutiva" ${ }^{1}$. Es el mundo (Welt) lo siempre pre-supuesto, lo evidente que simplemente se da, lo ya presente impersonal y evidente de suyo, aquello dentro de lo cual constante e invariablemente nos movemos.

\section{Niveles de lo psicológico}

Husserl ha dejado en claro que su "Psicología fenomenológica” ocupa un puesto único, especial e irremplazable al interior de la psicología. Pareciera que se ha alejado definitiva y completamente de los esfuerzos de Jaspers por elaborar una psicopatología fenomenológica. Sin embargo, la situación es bastante más compleja porque reconoce que la vida psicológica tiene varios estratos o niveles y la fenomenología se interesa sólo 
por un aspecto, central, pero no excluyente de los otros el de la conciencia. Sin embargo, Husserl dejó insinuados más que trabajados estos niveles, por lo que ellos deben ser inferidos a partir de anotaciones disperdigadas en diferentes textos de distintas épocas. Su discípulo Eugen Fink visualizó hacia donde se encaminaban los esfuerzos de Husserl en relación con la psicología hacia el final de su vida en su escrito La crisis de las ciencias europeas y la fenomenología trascendental, porque él colaboró en parte a redactar la versión definitiva a partir del manuscrito preparatorio ${ }^{32,62}$.

Apoyándose indirectamente en Fink, Hermann Drüe ha propuesto una jerarquía de tres niveles en psicología tomando como punto de referencia lo que sucede en la física: física práctica, física exacta y física pura ${ }^{40}$.

1) Psicología concreta o práctica. Como en fenomenología, concreta significa el estudio de la totalidad de los componentes de la realidad psicológica. Es la psicología psico-física. Comprende la vida psicológica íntegra con la inclusión del conjunto de sus elementos, dispositivos y mecanismos corporales y cerebrales subordinados. No se trata de la psicología naturalística o materialista, porque ésta tiene un presupuesto metafísico no probado, la existencia exclusiva de procesos orgánicos; por consecuencia, esta psicología no postula leyes propias o inherentes a la índole misma de lo psíquico, sino que todos los fenómenos mentales son reducidos a no ser sino simples epifenómenos de la materia.

2) Psicología efectivamente pura. De acuerdo a un proceso de abstracción que abarca todo lo orgánico, se dirige única y exclusivamente a lo psíquico mismo. Objeto de esta psicología son las vivencias y las variedades de vivencias, que deben ser estudiadas con supresión absoluta de los componentes somáticos o mecanismos fisicoquímicos. Se elimina completamente lo "natural", al igual que en las ciencias naturales teóricas se elimina íntegramente lo psíquico. Pero se permanece al interior de los hechos, realidades o sucesos psíquicos fácticos, sin incluir filosofías, ideologías, metafísicas foráneas.
3) Psicología pura eidética. Los temas a estudiar no son reales o no suceden en el espacio y tiempo objetivos, sino son esencias. Por ello es una ciencia a priori de las formas esenciales de un sujeto psíquico puro (no existente tampoco).

El mismo Drüe escoge ejemplos concretos sacados de la psiquiatría para justificar su propuesta de jerarquía ascendente al interior de la psicología. El primer nivel (Psicología psicofísica) estudia en la esquizofrenia las correlaciones psicofísicas entre alucinaciones auditivas y neurotransmisores cerebrales que están a su base condicionándolas. El segundo nivel (Psicología pura) puede adoptar una actitud abstractiva psicológica en relación al fenómeno del delirio y comprobar que papel juega en la vida mental de este o aquel enfermo esquizofrénico. En el tercer nivel (psicología eidética pura) se estudia el delirio al interior de "los atributos fundamentales de lo psíquico en sí", qué "estructura fundamental de subjetividad" configura, por tanto, se preocupa de la esencia de la alucinación y ya no más de las facticidades pertenecientes a la vida psíquica de un sujeto particular.

\section{Psicología fenomenológica y psicopatología fenomenológica}

La propuesta de Drüe es interesante, clara y estimulante, pero se sigue moviendo en el mismo tipo de insuficiencias o malas comprensiones que hemos anotado arriba. Así, el tercer nivel se corresponde bastante con la fenomenología de Jaspers, aunque éste se opuso a hablar de "eidética pura"-se puede comprobar gráficamente en el cuadro sinóptico que él escogió para diferenciar la percepción de la representación. Como el libro de Jaspers está estructurado metodológicamente, los niveles 1 y 2 aparecen investigados en profundidad en otros capítulos ${ }^{7}$.

Para finalizar, las conclusiones buscarán precisar lo que hasta aquí no ha sido posible: el lugar que puede tener la Psicología fenomenológica de Husserl al interior de la psicopatología en psiquiatría; mejor aún, si es posible formular una 
Psicopatología fenomenológica científica asentándose en aquella.

1) Husserl parte de las intuiciones originales, se atiene estrictamente a los hechos mismos, no construye teorías o hipótesis sin antes apoyarse en el suelo firme de los datos primarios incuestionables. Este "principio de todos los principios", según sus palabras, lo diferencia radicalmente de las ciencias psicológicas positivas ${ }^{63}$. Contrariamente en éstas, dice Bunge, "ya no se toman todos los datos por buenos: corregimos la experiencia, adoptando promedios o medianas y eliminando los datos que parecen irrazonables...., estamos más intolerantes o exigentes con los datos empíricos, pero más tolerantes para con las teorías" 64 .

2) La Psicología fenomenológica puede entregar con inmediatez y certidumbre originaria sólo las vivencias internas del propio fenomenólogo. El empleo metódico de las sucesivas técnicas de reducción, hacen posible la supresión de todo lo individual, real y el carácter de existente objetivo -permanece el "yo puro", la intimidad de la conciencia pura.

3) La Fenomenología psicopatológica, contrariamente, se refiere a otros yo los yo de los pacientes; las vivencias que surgen en la conciencia de éstos pueden convertirse en fenómenos sólo en tanto que otros yo al interior del yo del fenomenólogo. Esto quiere decir que se necesita llevar a cabo un regreso desde el yo puro del fenomenólogo, que es el primario, al yo del paciente, que es el derivado o secundario.

4) El yo puro del fenomenólogo posee una estructura especial. En torno a sí constituye lo que es específicamente propio (das Mir-Eigene), es la "esfera de la pertenencia". A diferencia de lo que le es propio al fenomenólogo, el otro yo del paciente es lo ajeno a él ${ }^{38}$.

5) Esta "esfera de pertenencia" es parte de la naturaleza del fenomenólogo, por ello Husserl la denomina "naturaleza en propiedad" (eigenheitliche Natur). Pues bien, el cuerpo constituye primariamente esta propiedad, cuerpo entendido como sistema orgánico de sensaciones y fenómenos cinestésicos que le permiten decir "yo puedo": yo puedo desplazarme, empujar, experimentar experiencias sensoriales. Eliminando todo lo ajeno, gracias al cuerpo integrado en la total "naturaleza en propiedad”, el fenomenólogo es un yo psicofísico con cuerpo, alma y yo personal.

6) La "esfera de pertenencia" del yo puro del fenomenólogo hace posible que éste, mediante su cuerpo, viva en el mundo, sin salir de sí conquiste el mundo, sea un ser en el mundo. Ahora bien, si algún enfermo mental aparece en su mundo, lo que le es presente de él es sólo su cuerpo, y ni siquiera como organismo viviente, sino como simple cuerpo físico. También solamente porque su propio cuerpo le es dado de manera original como organismo vivo y funcionante, el fenomenólogo puede hacer y hace una trasposición a partir de su propio cuerpo y la extiende al cuerpo ajeno y, al extenderla, este cuerpo ajeno se hace organismo. A este complejo acto de la conciencia Husserl lo llama "apresentación" (Appräsentation). Frente al objeto "cuerpo" que se hace presente del paciente, el fenomenólogo apresenta la parte de ese cuerpo que no está inmediatamente percibida por él, o sea, el "organismo". La presentación exige la apresentación; o sea, la conciencia tiende hacia el objeto apresentado, el organismo, con una intencionalidad no por mediada menos real que la del objeto presentado, el cuerpo ${ }^{65}$.

7) Esto significa, la semejanza entre el cuerpo del fenomenólogo y el cuerpo del paciente es el fundamento y motivo para concebir "por analogía” al cuerpo ajeno como otro organismo. Por trasposición extendida al organismo del otro, ese organismo a su vez se apresenta como ego ego como el yo del fenomenólogo, y a la vez alter: otro yo. Las palabras de Husserl son claras: "Lo que (en esta experiencia mía) puede ser presentado y justificado directamente, soy yo mismo o me pertenece en propiedad. Lo que, por el contrario, sólo puede serme dado mediante una experiencia indirecta o fundada, que no presenta el objeto mismo, sino que lo sugiere, y que a la vez verifica esta sugestión por concordancia interna, es el otro" 38 .

8) "El otro (el yo del paciente) remite a mí- 
mismo (el yo del fenomenólogo), es un reflejo de mí mismo (del fenomenólogo) y, sin embargo, hablando propiamente, no es un reflejo; es mi análogo (del fenomenólogo) y, sin embargo, tampoco es un análogo en el sentido habitual del término" ${ }^{38}$. Esta extraña frase, tomada de Husserl aunque poniéndole en paréntesis el tema que nos interesa, demuestra las serias dificultades que tuvo para poder resolver el problema del otro y su yo en nuestro caso, del paciente. Sus larguísimas lecciones y apuntes sobre la intersubjetividad sólo en parte arrojaron alguna luz, aunque de ningún modo definitiva, para nuestro tema ${ }^{66-68}$.

9) La Psicología fenomenológica puede fundar una Psicología positiva que sea justificada desde un punto de vista husserliano: que las intenciones se "verifiquen" o se "cumplan" (Erfüllung) mediante evidencias absolutas. Lo que no se puede afirmar es que pueda fundar una Psicopatología fenomenológica "originaria" o totalmente "evidente". El enfermo es siempre un otro, un yo apercibido por trasferencia - una trasposición extendida desde el cuerpo del fenomenólogo al cuerpo del paciente en tanto otro cuerpo, y de ahí a su intimidad. Esa intimidad es últimamente derivada, a posteriori, misterio y opacidad. Aunque el yo del enfermo co-existe con el yo del fenomenólogo es otro-yo, es un nos-otros con el yo del fenomenólogo ${ }^{69}$.

10) La conclusión parece inevitable. La psicopatología fenomenológica será siempre una ciencia de lo psíquico anormal inferida o secundaria, nunca apriórica o absoluta. Podrá buscar la jus- tificación de sus asertos de un modo cada vez más puro, pero sabiendo que no la alcanzará.

11) Es efectivo que la filosofía actual ha puesto en entredicho no sólo la imposiblidad de acceder de modo indubitable o inmediato a la interioridad del otro, sino que ha ido más allá. Ha rechazado de la manera más categórica que el "yo" o "sujeto" sea trasparente a sí, lo más cercano a sí mismo, el dato indubitable del que haya que partir para alcanzar la verdad primera. En frase de Ricoeur, en el mejor de los casos, hay una certidumbre de la conciencia inmediata, pero esta certidumbre no es un saber verdadero, un conocimiento auténtico o una comprensión absoluta de uno mismo ${ }^{70}$. Ni siquiera esta última aseveración la compartirían Nietzsche, Heidegger u Ortega, porque ellos dudan de la realidad de la conciencia; vale decir, para todos, el "yo" o "conciencia" es una construcción de la metafísica moderna y que, como construcción histórica, está en vías de desaparecer muy pronto ${ }^{71-73}$.

12) La respuesta de Husserl sería pronunciada con palabras fuertes e inequívocas: "El conocimiento puramente empírico... no es ciencia en sentido preciso. Eso solamente proporciona verdades puramente relativas a una situación. La filosofía, auténtica ciencia, apunta a verdades absolutas, definitivas, que vayan más allá de todas las relatividades. En ellas se determina el ser como es en sí mismo". Y agrega "como ciencia de la responsabilidad y justificación últimas [la fenomenología] renuncia al mundo y permanece en el Yo o Ego con la certeza de ser"74. 


\section{Resumen}

Antecedentes. La Psicología fenomenológica de Husserl es una disciplina destinada a jugar un papel importante en la psicopatología fenomenológica empírica. Objetivo. Centrarse en los supuestos filosóficos y psicológicos de la Psicología fenomenológica Método. Investigar las interpretaciones de Husserl acerca de la naturaleza de la Psicología fenomenológica para comprender los presupuestos de la psicopatología fenomenológica en psiquiatría. Resultados La Psicología fenomenológica es una ciencia a priórica, eidética, intuitiva, puramente descriptiva e intencional de lo psíquico, que permanece dentro de la actitud natural. Conclusiones. La Psicología fenomenológica está destinada a proporcionar los conceptos esenciales necesarios para dar un sentido a la investigación que se presenta con el título de Fenomenología psicopatológica.

Palabras clave: Husserl, psicopatología fenomenológica, fenomenología.

\section{Referencias}

1. Jaspers K. Die phänomenologische Forschungsrichtung in der Psychopathologie. Z gesam Neurol Psychiat 1912; 9: 391-408.

2. Jaspers K. Allgemeine Psychopathologie. Ein Leitfaden für Studierende, Ärzte und Psychologen. Berlin: Springer, 1913.

3. Kronfeld A. Über neuere pathopsychischphänomenologische Arbeiten. Zent f gesamt Neurol Psychiat 1922; XXVIII; 441-467.

4. Figueroa G. La "Psicopatología General" de K Jaspers en la actualidad: Fenomenología, comprensión y los fundamentos del conocimiento psiquiátrico. Rev Chil Neuro-Psiquiat 2000; 38: 167-186.

5. van den Berg JH.The phenomenological approach to psychiatry. Springfield: Thoma, 1955.

6. Figueroa G. La fenomenología en la psiquiatría. Rev Cien Sociales (Chile) 1983; 22: 207-229.

7. Jaspers K. Allgemeine Psychopathologie. 8 Aufl. Berlin: Springer; 1965.

8. Skinner BF. The behaviour of organisms. New York: Appleton Century Crofts; 1938.

9. Bolton D, Hill J. Mind, meaning, and mental disorder. The nature of causal explanation in psychology and psychiatry. Oxford: Oxford University Press, 1996.

10. Hempel CG. Philosophy of natural science. Englewood Cliffs, NJ: Prentice Hall; 1966.
11. Feighner JP, Robins E, Guze G, Woodruff RA, Winokur G, Muñoz R. Diagnostic criteria for use in psychiatric research. Arch Gen Psychiatry 1972; 26: 57-63.

12. Robins E, Guze SB. Establishment of diagnostic validity in psychiatric illness: its application to schizophrenia. Am J Psychiatry 1970; 126: 983-987.

13. Blashfield RK. The classification of psychopathology: neo-kraepelinean and quantitative approaches. New York: Plenum; 1984.

14. Kandel ER. A new intellectual framework for psychiatry. Am J Psychiatry 1998; 155: 457-469.

15. Figueroa G. Un marco de referencia nuevo para la psiquiatría: la mente encuentra al cerebro. I. Los fundamentos científicos y humanos. Rev Chil Neuro-Psiquiat 2002; 40: 307-320.

16. Figueroa G. Un marco de referencia nuevo para la psiquiatría: la mente encuentra al cerebro. II. Fundamentos históricos. Rev Chil Neuro-Psiquiat 2002; 40: 321-334.

17. American Psychiatric Association. Diagnostic and Statistical Manual of Mental Disorders, Third Edition. Washington, DC: American Psychiatric Association, 1980.

18. Blankenburg W. Grundlagenprobleme der Psychopathologie. Nervenartz 1978; 49: 140-146.

19. Janzarik W. Die Krise der Psychopathologie. Nervenartz 1976; 47: 73-80.

20. Andreasen N. DSM and the death of pheno- 
menology in America: An example of unintended consequences. Schizophr Bull 2007; 33: 108-112.

21. Spiegelberg H. Phenomenology in psychology and psychiatry. Evanston: Northwestern University Press; 1972.

22. Karlson H, Hamppinen M. Biological psychiatry and reductionism. Empirical findings and philosophy. Br J Psychiatry 1995; 167: 434-438.

23. Figueroa G. Delirio y realidad: Problemas de la psicopatología fenomenológica. Rev Chil NeuroPsiquiat 1989; 27: 92-99.

24. Straus E. Phenomenological psychology. New York: Basic Books, 1966.

25. de Koning AJJ, Jenner FA, editors. Phenomenology and psychiatry. London: Academic Press, 1982.

26. Blankenburg W. Psychiatrie und Philosophie. En : Kisker KP, Meyer JE, Müller C, Strömgren E, Hrsg. Psychiatrie der Gegenwart. Forschung und Praxis. Band I. Grundlagen und Methoden der Psychiatrie. Teil 1. 2. Auflage. Springer: Berlin, 1979, págs. 827875.

27. Husserl E. Logische Untersuchungen. Band I. Prolegomena zur reinen Logik. Husserliana XVIII. Den Haag: Martinus Nijhoff, 1975.

28. Husserl E. Logische Untersuchungen. Band II. Untersuchungen zur Phänomenologie und Theorie der Erkenntnis. Erster Teil. Husserliana XIX/1. Den Haag: Martinus Nijhoff, 1984

29. Husserl E. Logische Untersuchungen. Band II. Untersuchungen zur Phänomenologie und Theorie der Erkenntnis. Zweiter Teil. Husserliana XIX/2. Den Haag: Martinus Nijhoff, 1984

30. Jaspers K. Rechenschaft und Ausblick. Reden und Aufsätze. München: Piper; 1951.

31. Husserl E. Die Philosophie als strenge Wissenschaft. Logos 1910-1911; 1: 289-341.

32. Husserl E. Die Krisis der europäischen Wissenschaften und die transzendentale Phänomenologie. Husserliana VI. Den Haag: Martinus Nijhoff; 1962.

33. Kant I. Kritik der reinen Vernunft. ErsterTeil. Werke Band 3. Darmstadt: Wissenschaftliche Buchgesselschaft, 1968.

34. Husserl E. Ideen zu einer reinen Phänomenologie und phänomenologischen Philosophie. Husserliana III. Den Haag: Martinus Nijhoff; 1962.
35. Zubiri X. Cinco lecciones de filosofía. Madrid: Sociedad de estudios y publicaciones, 1963.

36. Jaspers K. Philosophie. 3 Bände. Berlin: Springer; 1932.

37. Descartes R. Discurso del método. Madrid: Revista de Occidente, 1974.

38. Husserl E. Cartesianische Meditationen und Pariser Vorträge. Husserliana I. Den Haag: Martinus Nijhoff; 1950.

39. Husserl E. Die Idee der Phänomenologie. Fünf Vorlesungen. Husserliana II. Den Haag: Martinus Nijhoff; 1950.

40. Drue H. Edmund Husserls System der phänomenologischen Psychologie. Berlin: de Gruyter; 1963.

41. Biemel W. Die entscheidende Phasen der Entfaltung von Husserls Philosophie. Z f philosoph Forschung 1959; 13: 187- 221.

42. Husserl E. Phänomenologische Psychologie. Vorlesungen Sommersemester 1925. Husserliana IX. Den Haag: Martinus Nijhoff; 1962.

43. Pinillos JL. La psicología fenomenológica. En: Homenaje a Xavier Zubiri. Tomo II. Madrid: Moneda y crédito, 1970. págs. 478-501.

44. Figueroa G. La psicología fenomenológica I. La crisis de la psicología. Rev Cien Sociales (Chile) 1985; 26-27: 231-246.

45. Brentano F. Psychologie vom empirischen Standpunkt. 3 Bände. Leipzig: Meiner, 1924-1928.

46. Gurwisch A. Edmund Husserl's conception of phenomenological psychology. En: Gurwisch A. Studies in phenomenology and psychology. Evanston: Northwestern University Press, 1966. págs. 76-112.

47. Ey H. La conscience. 2 édition. Paris: Presses Universitaires de France, 1968.

48. Searle JR. The mystery of consciousness. New York: New York Review of Books, 1997.

49. Searle JR. Intentionality: An essay in the philosophy of mind. Cambridge: Cambridge University Press, 1983.

50. Dennett DC. Consciousness explained. New York: Little, Brown, 1991.

51. Dennett DC. The intentional stance. Cambridge: MIT Press, 1987.

52. Chalmers DJ. The conscious mind. In search of a fundamental theory. New York: Oxford University 
Press, 1996.

53. Hameroff S, Kaszniak A, Scott A, comp. Toward a science of consciousness. Cambridge: MIT Press, 1996.

54. Sartre J-P. Une idée fondamental de la phénomenologie de Husserl: l'intentionalité. En: Sartre JP. Situatons I. Paris: Gallimard, 1959. págs. 25-29

55. Kockelmans J. Edmund Husserl's phenomenological psychology. A historical-critical study. Pittsburgh: Duquesne University Press; 1967.

56. Kockelmans J. Husserl's original view on phenomenological psychology. En: Kockelmans J, editor. Phenomenology. The philosophy of Edmund Husserl and its interpretation. New York: Doubleday \& Company, 1967. págs. 418-449.

57. Szilasi E. Einführung in die Phänomenologie Edmund Husserls. Tübingen: Niemeyer, 1959.

58. Blankenburg W. Phänomenologie der LebensweltBezogenheit des Menschen und Psychopathologie. En: Grathoff R, Waldenfels B, Hrsg. Sozialität und Intersubjektivität. München: Fink, 1983. págs. 182207.

59. Biemel W. Reflexionen zur Lebenswelt-Thematik. En: Biemel W, Hrsg. Phänomenologie heute. Den Hagg: Nijhoff, 1972. págs.49-77.

60. Biemel W. Husserls Encyclopaedia-BritannicaArtikel und Heideggers Anmerkungen dazu. En: Noack H, Hrsg. Husserl. Darmstadt: Wissenschaftliche Buchgesellschaft, 1973. págs. 282-315.

61. Husserl E. Ideen zu einer reinen Phänomenologie und phänomenologischen Philosophie. Zweitestes Buch. Phänomenologishen Untersuchungen zur Konstitution.. Husserliana IV. Den Haag: Martinus Nijhoff; 1952.

62. Fink E. Studien zur Phänomenologie (1930-1939).
Den Haag: Nijhoff, 1966.

63. Husserl E. Erste Philosophie (1923-1924). Erster Teil. Kritische Ideengeschichte. Husserliana VII. Den Haag: Martinus Nijhoff, 1959.

64. Bunge M. Epistemología. Barcelona: Ariel, 1985.

65. Husserl E. Ideen zu einer reinen Phänomenologie und phänomenologischen Philosophie. Drittes Buch. Die Phänomenologie und die Fundamente der Wissenschaften. Husserliana V. Den Haag: Martinus Nijhoff; 1984.

66. Husserl E. Zur Phänomenologie der Intersubjektivität. Texte aus dem Nachla ${ }^{2}$. I: 19051920. Husserliana XIII. Den Haag: Martinus Nijhoff; 1973.

67. Husserl E. Zur Phänomenologie der Intersubjektivität. II: 1921-1928. Husserliana XIV. Den Haag: Martinus Nijhoff; 1973.

68. Husserl E. Zur Phänomenologie der Intersubjektivität. III: 1929-1935. Husserliana XV. Den Haag: Martinus Nijhoff; 1973.

69. Laín Entralgo P. Teoría y realidad del otro. 2 tomos. Madrid: Revista de Occidente, 1961.

70. Ricoeur P. Du texte à l'action. Essais d'herméneutique II. París: Seuil; 1986.

71. Ortega y Gasset J. La idea de principio en Leibniz y la evolución de la teoría deductiva. OC VIII 1948; p. 59-356.

72. Heidegger M. Sein und Zeit. 10. Aufl. Tübingen: Niemeyer; 1963.

73. Nietzsche F. Aus dem Nachla ${ }^{2}$ der Achtzigerjahre. Werke in drei Bänden III. München: Hanser, 1966. p. 415-925.

74. Husserl E. Phänomenologie und Anthropologie. Philosophy and phenomenological research 19631964; 24: 355-375.
Correspondencia:

Gustavo Figueroa Cave

Casilla 92-V

E-mail: gufigueroa@vtr.net

gusta.figueroa@hotmail.com 